\title{
Extent of Bilateral Neuronal Network Reorganization and Functional Recovery in Relation to Stroke Severity
}

\author{
Maurits P.A. van Meer, ${ }^{1,2 \star}$ Willem M. Otte, ${ }^{1,3 \star}$ Kajo van der Marel, ${ }^{1 \star}$ Cora H. Nijboer, ${ }^{4}$ Annemieke Kavelaars, ${ }^{4}$ \\ Jan Willem Berkelbach van der Sprenkel, ${ }^{2}$ Max A. Viergever, ${ }^{1}$ and Rick M. Dijkhuizen ${ }^{1}$ \\ ${ }^{1}$ Biomedical MR Imaging and Spectroscopy Group, Image Sciences Institute, University Medical Center Utrecht, 3584 CX Utrecht, The Netherlands, \\ ${ }^{2}$ Department of Neurosurgery, Rudolf Magnus Institute of Neuroscience, University Medical Center Utrecht, 3584 CX Utrecht, The Netherlands \\ ${ }^{3}$ Department of Pediatric Neurology, Rudolf Magnus Institute of Neuroscience, University Medical Center Utrecht, 3584 EA Utrecht, The Netherlands, and \\ ${ }^{4}$ Laboratory of Neuroimmunology and Developmental Origins of Disease, University Medical Center Utrecht, 3584 EA Utrecht, The Netherlands
}

Remodeling of neuronal structures and networks is believed to significantly contribute to (partial) restoration of functions after stroke. However, it has been unclear to what extent the brain reorganizes and how this correlates with functional recovery in relation to stroke severity. We applied serial resting-state functional MRI and diffusion tensor imaging together with behavioral testing to relate longitudinal modifications in functional and structural connectivity of the sensorimotor neuronal network to changes in sensorimotor function after unilateral stroke in rats. We found that gradual improvement of functions is associated with wide-ranging changes in functional and structural connectivity within bilateral neuronal networks, particularly after large stroke. Both after medium and large stroke, brain reorganization eventually leads to (partial) normalization of neuronal signal synchronization within the affected sensorimotor cortical network (intraregional signal coherence), as well as between the affected and unaffected sensorimotor cortices (interhemispheric functional connectivity). Furthermore, the bilateral network configuration shifts from subacutely increased "small-worldness," possibly reflective of initial excessive neuronal clustering and wiring, toward a baseline small-world topology, optimal for global information transfer and local processing, at chronic stages. Cortical network remodeling was accompanied by recovery of initially disrupted structural integrity in corticospinal tract regions, which correlated positively with retrieval of sensorimotor functions. Our study demonstrates that the degree of functional recovery after stroke is associated with the extent of preservation or restoration of ipsilesional corticospinal tracts in combination with reinstatement of interhemispheric neuronal signal synchronization and normalization of smallworld cortical network organization.

\section{Introduction}

Stroke is the main cause of adult disability in the world. Nevertheless, most patients exhibit a certain degree of restoration of sensorimotor function after acute neurological deficits. This spontaneous functional recovery has been attributed to brain plasticity, i.e., structural and functional adaptation of neuronal elements and networks. Yet, it remains incompletely understood to what extent the poststroke brain reorganizes locally and glob-

Received July 18, 2011; revised Jan. 30, 2012; accepted Feb. 8, 2012.

Author contributions: M.P.A.v.M., J.W.B.v.d.S., M.A.V., and R.M.D. designed research; M.P.A.v.M., W.M.0., K.v.d.M., and C.H.N. performed research; W.M.O., K.v.d.M., and A.K. contributed unpublished reagents/analytic tools; M.P.A.v.M., W.M.O., K.v.d.M., and A.K. analyzed data; M.P.A.v.M. and R.M.D. wrote the paper.

The research leading to these results has received funding from the Alexandre Suerman program of the University Medical Center Utrecht, Utrecht University's High Potential program, the Netherlands Organization for Scientific Research (NWO), and the European Union's Seventh Framework Programme (FP7/2007-2013) under grant agreements 201024 and 202213 (European Stroke Network). We thank Gerard van Vliet, Annette van der Toorn, René Zwartbol, and Ward Jennekens for technical assistance.

${ }^{*}$ M.P.A.v.M, W.M.O., and K.v.d.M. contributed equally to this work.

The authors declare no competing financial interests.

Correspondence should be addressed to Rick M. Dijkhuizen or Maurits P.A. van Meer, Image Sciences Institute, University Medical Center Utrecht, Yalelaan 2, 3584 CM Utrecht, The Netherlands. E-mail: rick@isi.uu.nl or maurits@invivonmr.uu.nl.

DOI:10.1523/JNEUROSCI.3662-11.2012

Copyright $\odot 2012$ the authors $\quad 0270-6474 / 12 / 324495-13 \$ 15.00 / 0$ ally, in what manner structural and functional modifications of neuronal circuitry are interrelated, and to what degree this may lead to functional improvement. Evidently, elucidation of the neuronal mechanisms that underlie poststroke recovery may guide development of therapeutic strategies that enhance these processes.

During the last two decades, functional imaging studies have provided evidence of cerebral reorganization by displaying shifts of stimulus, or task-induced activation patterns in the affected (ipsilesional) and opposite (contralesional) brain hemispheres after clinical and experimental stroke (Calautti and Baron, 2003; van Meer and Dijkhuizen, 2010). More recently, measurement of spontaneous low-frequency blood oxygenation level-dependent (BOLD) fluctuations with resting-state functional MRI (fMRI) has revealed changes in interhemispheric synchronization of baseline neuronal signaling, which correlated with behavioral outcome (He et al., 2007; Carter et al., 2010; van Meer et al., 2010b). Furthermore, resting-state fMRI combined with network analysis based on graph theory demonstrated a shift toward a more random topology of the motor execution network in recovering stroke patients (Wang et al., 2010). Such alterations in the organization of functional brain networks are highly dependent on the configuration and flexibility (i.e., plasticity) of underlying neuronal architecture. Various forms of structural plasticity of neurons, such as changes in dendritic morphology, axonal 
sprouting, and synaptogenesis (Carmichael, 2003; Nudo, 2007; Murphy and Corbett, 2009), may lead to critical adjustments of local structural connectivity. In addition, studies that have applied diffusion tensor imaging (DTI) have reported widespread white matter remodeling based on the observation of increased fractional anisotropy (FA) of water diffusion in white matter fiber tracts (Jiang et al., 2006; Wang et al., 2006; van der Zijden et al., 2008b; Schaechter et al., 2009), which may contribute to improvement of connectivity between remote brain areas.

Current data clearly establish the adult brain's capacity to reorganize after ischemic injury. Yet, despite the accumulating evidence of alterations in neuronal circuits, it remains unclear whether and to what extent regaining of function after stroke is dependent on specific perilesional adaptation or large-scale brain remodeling. Furthermore, the association between functional and structural modifications in relation to the degree of injury in whole functional networks is still unknown. Therefore, we combined serial resting-state fMRI, DTI, and network analysis to characterize the dynamics of functional and structural alterations at a network level in sensorimotor cortices and tracts in relation to different sensorimotor recovery patterns after experimental stroke. We hypothesized that gradual recovery, particularly after large stroke, is associated with extensive functional and structural remodeling in the whole bilateral sensorimotor network.

\section{Materials and Methods}

Animal handling. All animal procedures were approved by the Animal Experiments Committee of the University Medical Center Utrecht and Utrecht University, and experiments were performed in accordance with the guidelines of the European Communities Council Directive.

Twenty-four young adult male Sprague Dawley rats, weighing 280$320 \mathrm{~g}$, were included in the study. Fourteen rats underwent transient focal cerebral ischemia. Ten rats served as age-matched controls. Behavioral testing and MRI measurements were done at $2 \mathrm{~d}$ before $(=$ day -2$)$ and at $3,7,21,49$, and $70 \mathrm{~d}$ after stroke. Control rats were scanned with the same time intervals as rats poststroke.

Rats were anesthetized with $4 \%$ isoflurane for endotracheal intubation, followed by mechanical ventilation with $2 \%$ isoflurane in air $/ \mathrm{O}_{2}$ (2/1) during surgery, MRI, and electroencephalogram (EEG) recordings. Blood oxygen saturation and heart rate were continuously monitored and body temperature was maintained at $37.0 \pm 0.5^{\circ} \mathrm{C}$. Furthermore, during MRI, expired $\mathrm{CO}_{2}$ was continuously monitored with a capnograph, and ventilation volume or rate was adjusted when necessary.

Stroke model. Experimental stroke was induced by 90 min occlusion of the right middle cerebral artery with an intraluminal filament as previously described (Longa et al., 1989; van Meer et al., 2010b).

Behavioral testing. Sensorimotor function after stroke was measured using the sensorimotor performance score on a scale of 0 to -20 points (van der Zijden et al., 2008a). Scoring was based on (1) spontaneous activity during $1 \mathrm{~min}$, (2) gait disturbances such as circling toward the paretic side, (3) degree or absence of pathological postural reflexes when held by the tail, (4) degree of asymmetry in resistance between left and right lateral push, (5) degree of asymmetry in grasping strength onto a rod between left and right forepaw, and (6) degree or absence of forelimb placing onto a table rim after stimulation of the vibrissae.

MRI. MRI measurements were conducted on a $4.7 \mathrm{~T}$ horizontal bore MR system (Varian). A Helmholtz volume coil ( $90 \mathrm{~mm}$ diameter) and an inductively coupled surface coil (25 $\mathrm{mm}$ diameter) were used for signal excitation and detection, respectively. Rats were placed in an MRcompatible stereotactic holder and immobilized with earplugs and a tooth-holder. Multi-echo, multislice $\mathrm{T}_{2}$-weighted MRI (repetition time $[\mathrm{TR}] /$ echo time $[\mathrm{TE}]=3600 / 15 \mathrm{~ms}$; echo train length $=12 ; 191 \mathrm{~mm}$ coronal slices; field of view $[\mathrm{FOV}]=32 \times 32 \mathrm{~mm}^{2}$; acquisition matrix $=$ $256 \times 128$; voxel resolution $=0.25 \times 0.25 \times 1.0 \mathrm{~mm}^{3}$ ) was done to determine the extent of the ischemic lesion. For registration purposes, gradient echo $3 \mathrm{D}$ MRI $\left(\mathrm{TR} / \mathrm{TE}=6 / 2.6 \mathrm{~ms}\right.$; flip angle $=40^{\circ} ; \mathrm{FOV}=60 \times$
$40 \times 40 \mathrm{~mm}^{3}$; acquisition matrix $=256 \times 128 \times 128$; voxel resolution $=$ $0.23 \times 0.31 \times 0.31 \mathrm{~mm}^{3}$ ) was included. DTI was performed with a four-shot spin-echo, echo planar imaging (EPI) sequence (TR/TE $=$ $3500 / 26 \mathrm{~ms} ; 250.5 \mathrm{~mm}$ axial slices; FOV $=32 \times 32 \mathrm{~mm}^{2}$; acquisition matrix $=64 \times 64$; voxel resolution $=0.5 \times 0.5 \times 0.5 \mathrm{~mm}^{3}$; diffusionweighted images in 50 directions with $b=1250 \mathrm{~s} / \mathrm{mm}^{2}$; two images without diffusion-weighting $(b=0)$; number of averages $=4)$. BOLD resting-state fMRI was conducted during $10 \mathrm{~min}$ with a gradient echo EPI sequence $\left(\mathrm{TR} / \mathrm{TE}=500 / 19 \mathrm{~ms}\right.$; flip angle $=35^{\circ}$; seven $1.5 \mathrm{~mm}$ coronal slices; FOV $=32 \times 32 \mathrm{~mm}^{2}$; acquisition matrix $=64 \times 64$; voxel resolution $=0.5 \times 0.5 \times 1.5 \mathrm{~mm}^{3} ; 1200$ images $)$.

Before resting-state fMRI, end-tidal isoflurane was reduced to $1 \%$ for $10 \mathrm{~min}$. Animals remained sufficiently anesthetized during this period and we observed no signs of discomfort.

Histology. Animals were killed after the final MRI session, followed by transcardial perfusion-fixation with $4 \%$ paraformaldehyde. Brains from a subset of animals $(N=6)$ were extracted and embedded in paraffin. Hematoxylin and eosin (H\&E) staining and Luxol Fast Blue staining were performed on coronal sections of $8 \mu \mathrm{m}$. Sections were first deparaffinized and hydrated to $95 \%$ ethyl alcohol, followed by H\&E staining procedure or overnight incubation in $0.1 \%$ Luxol Fast Blue at $56^{\circ} \mathrm{C}$. Thereafter sections were washed in 95\% ethyl alcohol, rinsed in distilled water, differentiated in lithium carbonate solution for $30 \mathrm{~s}$ followed by $70 \%$ ethyl alcohol for $30 \mathrm{~s}$, and finally rinsed in distilled water.

EEG recording. In a separate group of rats EEGs were recorded epidurally from the left and right primary motor cortices according to the rat stereotaxic atlas (Paxinos and Watson, 2005) at $2 \mathrm{~d}$ before, and 3 and $21 \mathrm{~d}$ after stroke $(N=8)$. Before $E E G$, end-tidal isoflurane was reduced to $1 \%$ for $10 \mathrm{~min}$. EEG signals were then acquired during $10 \mathrm{~min}$ using a homebuilt 10-channel amplifier at a sampling rate of $1000 \mathrm{~Hz}$ per channel, and bandpass filtered between 0.1 and $250 \mathrm{~Hz}$. An analog-to-digital converter (NI USB-6211; DAQ) and LabWindows software (National Instruments) were used for data acquisition. The Fisher-transformed correlation coefficients $\left(z^{\prime}=\ln ((1+r) /(1-r)) / 2\right)$ between temporal fluctuations of the EEG signals from left and right cortices were calculated for the delta $(1-5 \mathrm{~Hz})$, theta $(5-8 \mathrm{~Hz})$, alpha $(8-15 \mathrm{~Hz})$, beta $(15-30$ $\mathrm{Hz})$, and gamma $(30-250 \mathrm{~Hz})$ frequency bands. Three rats died before day 3 due to severe stroke-induced cachexia. The five remaining rats displayed exclusively subcortical stroke lesions on $\mathrm{T}_{2}$-weighted MRI at $3 \mathrm{~d}$ poststroke.

Image registration. Nonrigid image registration was performed with Elastix (Klein et al., 2010) (http://elastix.isi.uu.nl) to align gradient echo $3 \mathrm{D}$ images with $\mathrm{a} \mathrm{T}_{2}$-weighted anatomical rat brain template. Affine-only registration within each dataset was performed for the $\mathrm{T}_{2}$-weighted MRI and resting-state fMRI scans.

Lesion segmentation. Stroke lesions characterized by $\mathrm{T}_{2}$ prolongation were manually outlined on $\mathrm{T}_{2}$ maps by two independent neuroscientists. The overlapping area was assigned as lesioned tissue.

Signal coherence and functional connectivity analysis. Preprocessing of resting-state fMRI data included spatial smoothing with an isotropic Gaussian kernel of $1.0 \mathrm{~mm}$ full-width at half-maximum, rigid-body motion correction using FMRIB's Linear Image Registration Tool (FMRIB FSL; http://www.fmrib.ox.ac.uk/fsl) and linear regression against (1) rigid-body realignment parameters, (2) derivatives of realignment parameters, and (3) global mean signal to minimize potential BOLD fluctuations of non-neuronal origin and to increase the specificity of neuronal functional connectivity measurements (Fox et al., 2009; Weissenbacher et al., 2009). Blocks of nine scans with $>0.05 \mathrm{~mm}$ translational or $>2.5^{\circ}$ rotational motion were discarded from further analysis. The same number of data points (i.e., 400 scans) was removed in corresponding time blocks for all datasets. Low-frequency BOLD fluctuations were obtained from the functional time series with a bandpass filter at $0.01<$ $f<0.1 \mathrm{~Hz}$.

Preprocessed resting-state fMRI data at the first time point from 20 animals were temporally concatenated to create a single $4 \mathrm{D}$ dataset. Next, probabilistic independent component analysis (Beckmann et al., 2005) was performed, using Multivariate Exploratory Linear Optimized Decomposition into Independent Components software (FMRIB FSL; http://www. fmrib.ox.ac.uk/fsl), to extract a group-averaged functional network that 
included the bilateral sensorimotor cortex. This data-driven approach allows unbiased identification of whole functional networks from included animals, without the need to coregister to an extrinsic rat brain atlas to select diverse brain regions that are assumed to be part of a larger functional network (Hutchison et al., 2010; Jonckers et al., 2011). We found that analysis with seven components yielded a component of which the anatomical pattern most ideally matched with the bilateral sensorimotor cortices (see Fig. 2). Thresholding at $Z=4$ provided left and right sensorimotor cortical areas, which were used as regions of interest (ROIs) for further analyses. Voxels inside the lesion territory $(0 \pm 2 \%$ and $12 \pm 8 \%$ of total bilateral ROI volume

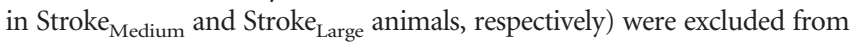
analyses. We used dual regression to back-reconstruct the group-level components at an individual level (Zuo et al., 2010). Voxel-by-voxel incidence maps were created using the back-reconstructed individual components and overlaid on a $\mathrm{T}_{2}$-weighted anatomical rat brain template.

Whole-brain functional connectivity maps were obtained by voxelwise calculation of the Fisher-transformed correlation coefficient, $z^{\prime}$, with the mean time series signal from the left sensorimotor cortex as reference. Group mean functional connectivity maps with left sensorimotor cortex as seed region were constructed using one-sample $t$ testing. Functional connectivity difference maps between time points were constructed using two-sample paired $t$ testing. The $P$ values were projected on a $\mathrm{T}_{2}$-weighted anatomical rat brain template and corrected for multiple-comparisons using the AlphaSim command in AFNI (Cox, 1996; Ward, 2000).

Intraregional signal coherence and interregional functional connectivity were calculated as $z^{\prime}$ between time courses of low-frequency BOLD fMRI signals. Interregional functional connectivity was determined as $z^{\prime}$ between the mean low-frequency BOLD signal fluctuations in left and right sensorimotor cortices. Intraregional signal coherence in left and right sensorimotor cortices was calculated as the mean $z^{\prime}$ between the low-frequency BOLD signal fluctuations of each voxel within the ROI and the average low-frequency BOLD signal time series of that ROI (Wang et al., 2011).

Graph-based network analysis. For each resting-state fMRI dataset a weighted graph $G=(V, W)$ was constructed, with $V$ as the collection of $N$ voxels (mean \pm SD $=189 \pm 19$ ) in left and right sensorimotor cortices, and $W$ as the collection of edge weights $w_{i j}$, i.e., $z^{\prime}$ between any pair of voxel time series $i$ and $j$. Self-connections and negative edge weights were excluded. The local and global graph structures were quantified via the weighted undirected clustering coefficient $C$ (Fagiolo, 2007) and the weighted shortest path length $L$ (Stam et al., 2009) using the C++ Boost Graph Library (http://www.boost.org).

$C$ was defined as the mean of the local clustering coefficients of all connected nodes in the entire graph as follows:

$$
C=N^{-1} \sum_{i=1}^{N} C_{i},
$$

with the clustering coefficient for node $i$ as follows:

$$
C_{i}=\frac{\sum_{j \neq i} \sum_{h \neq(i, j)} w_{i j}^{\frac{1}{3}} w_{i h}^{\frac{1}{3}} w_{j h}^{\frac{1}{3}}}{d_{i}\left(d_{i}-1\right)},
$$

taking into account weights of all edges in a triangle, and excluding weights not participating in any triangle.

$L$ was defined as the mean geodesic length over all couples of nodes in the entire graph as follows:

$$
L=N(N-1) \mid \sum_{i=1, j \neq i}^{N} \frac{1}{l_{i j}^{w}}
$$

where $l_{i j}^{w}=\min \left(\operatorname{sum}\left(d_{i j}\right)\right)$ and $d_{i j}=1 / w_{i j}$.

Here, the weighted shortest path length, $l_{i j}^{w}$, between any pair of nodes $i$ and $j$ in the graph indicated the minimum value of the sum of transformed weights $d_{i j}$ (i.e., functional distance) over all possible paths. The harmonic mean was used to handle infinite path lengths between discon- nected edges (i.e., $1 / \infty \rightarrow 0$ ). In this way, the average weighted shortest path length $L$ resembled the global efficiency measure (Latora and Marchiori, 2001).

For each resting-state fMRI dataset, $C$ and $L$ were normalized based on 10 rewired surrogate networks (Maslov and Sneppen, 2002). Normalized weighted $C$ and $L$ ratios were defined as follows: $\gamma=C /<C_{\text {surrogate }}>$ and $\lambda=$ $L /<L_{\text {surrogate }}>$. Small-worldness, $\sigma$, of the bilateral sensorimotor cortical network was defined as $\gamma / \lambda$, according to Humphries and Gurney (2008).

In addition to the graph-based analysis of the current resting-state fMRI dataset, we retrospectively calculated $\gamma, \lambda$, and $\sigma$ of the bilateral sensorimotor cortex from resting-state fMRI data from our previous study (van Meer et al., 2010b). Functional connectivity, signal coherence, and graph-based network parameters were calculated from combined bilateral intact sensorimotor cortical regions, i.e., the hindlimb and forelimb region of the primary somatosensory cortex, primary motor cortex, and secondary motor cortex according to the rat brain atlas (Paxinos and Watson, 2005), in rats with only subcortical lesions $(N=5)$, and in rats with large lesions involving both subcortical and cortical tissue $(N=9)$, at poststroke days 3, 7, 21, and 70 .

Analysis of white matter integrity. FA maps were derived from DTI data using multivariate fitting and diagonalization (Basser and Pierpaoli, 1996), and nonrigidly registered to a common rat brain FA template from forty control animals, coregistered to the stereotaxic rat brain atlas (Paxinos and Watson, 2005).

Localized statistical testing of whole-brain FA data was performed using tract-based spatial statistics (Smith et al., 2007). A white matter skeleton was created from all registered FA maps by averaging, thresholding at 0.2 , and thinning to the centers of all tracts common to all subjects. Individual FA values were projected onto this mean white matter skeleton for subsequent voxelwise statistical analysis performed using nonparametric, permutation-based $t$ testing [false discovery ratecorrected (Nichols and Hayasaka, 2003)] between time points for each group. Statistically significant differences were thresholded at $p<0.05$, and projected onto a rat brain FA template.

White matter regions based on the coregistered stereotaxic rat brain atlas and outside the stroke lesion area were delineated on the FA rat brain template for ROI analysis. These included the corpus callosum, the left and right cerebral peduncles, and the left and right internal capsules (where the right internal capsule was divided in an anterior and a posterior part) (see Fig. 5C). If necessary, ROI position was manually corrected to adjust for stroke-induced tissue deformation.

Mean gray value intensities on whole-brain photographs of the Luxol Fast Blue-stained histological sections were measured in the same white matter regions as described above (manually delineated by M.P.A.v.M., an expert in rat neuroanatomy), using ImageJ software (NIH Image).

Statistical analyses. A Mann-Whitney $U$ test was used to evaluate differences in sensorimotor performance scores. Repeated-measures linear mixed model analysis (Laird and Ware, 1982) with fixed effects "group," "time" and "group $\times$ time," and random effect "subject" was used to assess changes in interhemispheric correlations of EEG signals, restingstate fMRI-based signal coherence, functional connectivity and network parameters, and DTI-based FA between groups and over time. Post hoc Tukey's testing was used to correct for multiple-comparisons. The linear mixed model analysis was also used to calculate correlation coefficients $(r)$ between changes in white matter integrity, i.e., FA and intercortical functional connectivity between successive time points, and between normalized FA and Luxol Fast Blue staining ((individual value - mean)/ $\mathrm{SD})$ in white matter ROIs, and between ranked changes in sensorimotor function versus ranked changes in (1) functional connectivity, (2) signal coherence, (3) network parameters $(\gamma, \lambda$, and $\sigma$ ), and (4) FA. To assess to what extent eventual functional outcome is related to one or more of the subacutely acquired MRI-based parameters, we calculated Spearman's rank correlation coefficients $(\rho)$ between functional connectivity, signal coherence, network parameters, FA, sensorimotor performance score, or lesion volume at $3 \mathrm{~d}$ poststroke and sensorimotor performance score at $70 \mathrm{~d}$ poststroke.

Discrete sensorimotor performance scores are presented as medians, whereas other continuous data are reported as mean $\pm \mathrm{SD}$, unless mentioned otherwise. All statistical analyses were performed in $R$ (Ihaka and 
Gentleman, 1996) using the stats package for the Mann-Whitney $U$ and Spearman's rank correlation tests, and the nlme and lme 4 packages for the linear mixed model analyses, where $p<0.05$ was considered significant.

\section{Results}

\section{Stroke lesion severity and} experimental groups

Fourteen rats underwent $90 \mathrm{~min}$ occlusion of the right middle cerebral artery. One animal died acutely due to strokeinduced cachexia. All other animals completed the protocol up to 10 weeks poststroke. In correspondence with our previous study (van Meer et al., 2010b), we identified two basic ischemic lesion patterns on $\mathrm{T}_{2}$ maps (characterized by $\mathrm{T}_{2}$ prolongation): rats with medium-sized lesions in primarily subcortical tissue, with occasional involvement of ventrolateral cortical tissue (group Stroke Medium; $_{\text {m }}=$ 5), and rats with large lesions involving subcortical and ventrolateral and dorsolateral cortical tissue (group Stroke Large; $N=8$ ) (Fig. 1A). Ten control rats formed group Control.

\section{Sensorimotor function}

Sensorimotor function, characterized by the sensorimotor performance score, was reduced subacutely after stroke, with a significantly lower score for Stroke Large rats than Stroke $_{\text {Medium }}$ rats at poststroke days 3,7 , and $21(p<0.05)$ (Fig. $1 B)$. Sensorimotor performance scores improved between days 3 and 70 after stroke, and were not significantly different between Stroke Medium $_{\text {and }}$ Stroke Large $_{\text {at days }}$ 49 and 70 after stroke.

\section{Signal coherence and functional connectivity in sensorimotor cortices}

To identify the baseline functional network that includes the bilateral sensorimotor cortices, we applied probabilistic independent component analysis of mean low-frequency BOLD signals acquired from all first resting-state fMRI scans in groups Stroke $_{\text {Large, }}$ Stroke $_{\text {Medium }}$, and Control. Temporal concatenation and probabilistic independent component analysis of all time series provided different network components with synchronized resting-state fMRI signals (Fig. 2). Visual inspection revealed a network component that was anatomically characterized by the left and right primary and secondary somatosensory and primary motor cortices according to the rat brain atlas (Paxinos and Watson, 2005) at threshold level $Z=$ 4 , which was most apparent for probabilistic independent component analysis based on seven components (Fig. 2, component five). The left and right parts of the encompassed cortical area were used as sensorimotor cortical ROIs for subsequent analyses of intracortical signal coherence and interhemispheric functional connectivity. In addition, similar analyses were done on an alternative network, for which we selected the component that encompassed the bilateral cingulate and secondary motor cortices as well as the bilateral dorsal thalamus (Fig. 2, component four).

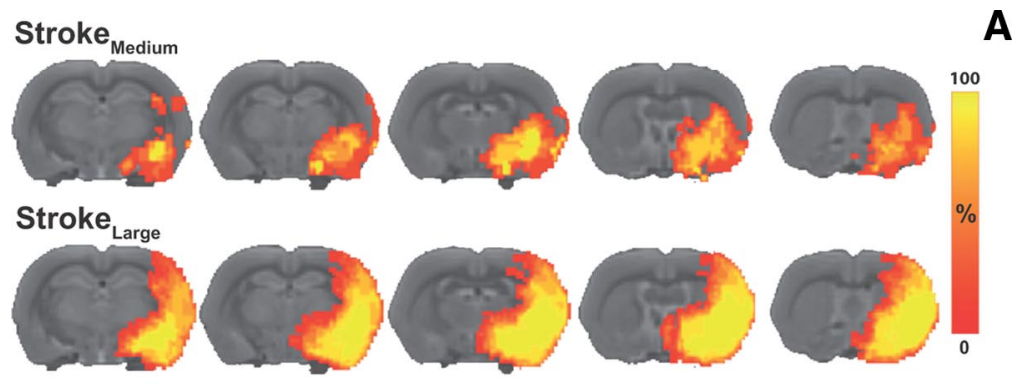

A

B

Figure 1. Lesion incidence and sensorimotor performance. $A$, Maps of the incidence of right-sided ischemic lesions characterized by prolonged $T_{2}$ at 10 weeks poststroke, overlaid on consecutive coronal slices from a multislice $T_{2}$-weighted anatomical rat brain template. Animals were divided in two stroke groups based on the extent of the lesion. Medium stroke (Stroke Medium $_{\text {) }}$ was

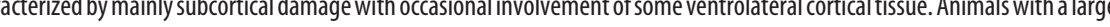
in the Stroke ${ }_{\text {Medium }}$ (green; $N=5$ ) and Stroke Large $($ red; $N=8)$ groups.

Statistical maps of functional connectivity of the left (contralesional) sensorimotor cortex (Fig. $3 A$ ) with the rest of the brain, clearly illustrated that the strong baseline interhemispheric functional connectivity between the left and right sensorimotor cortices was lost at $3 \mathrm{~d}$ after stroke in the Stroke $_{\text {Medium }}$ and Stroke Large $_{\text {groups }}$ (Fig. $3 B$ ). To delineate the pattern of functional connectivity alterations when animals recovered from stroke, we mapped the areas where functional connectivity significantly changed from its level at day 3 (Fig. 3C). The pattern of changes in functional connectivity with the left, contralesional sensorimotor cortex was clearly different between the Stroke $_{\text {Medium }}$ and Stroke Large $_{\text {groups. In Stroke }}$ Medium animals, functional connectivity with left sensorimotor cortex had increased in the right, ipsilesional sensorimotor cortex 21, 49, and $70 \mathrm{~d}$ after stroke, as compared with the loss after $3 \mathrm{~d}$, with a complete return to baseline levels after day 70 (Fig. $4 A$ ). Such recovery of intercortical functional connectivity was not seen in the Stroke $_{\text {Large }}$ group; intercortical functional connectivity somewhat improved after the dip at day 7, but remained significantly lowered at all time points after stroke (Fig. 4A). The same pattern was observed for interhemispheric functional connectivity in the bilateral cingulatethalamic network (data not shown).

Intraregional signal coherence in right, ipsilesional sensorimotor cortex was also significantly reduced at 3 and $7 \mathrm{~d}$ poststroke in Stroke $_{\text {Medium }}$ and Stroke $\mathrm{Large}_{\text {( }}(p<0.05$ vs baseline, days 3 and 7 , or controls, day 7) (Fig. 4B). This was restored in Stroke Medium $_{\text {animals }}$ 
A
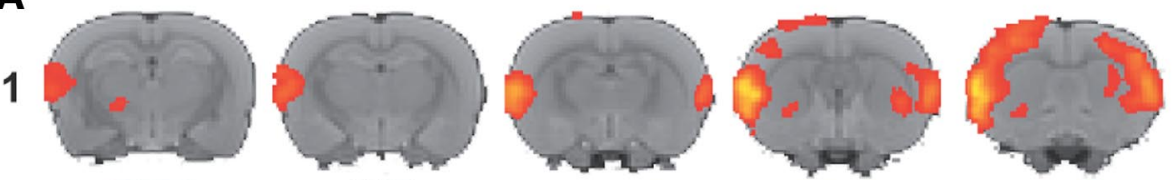

2
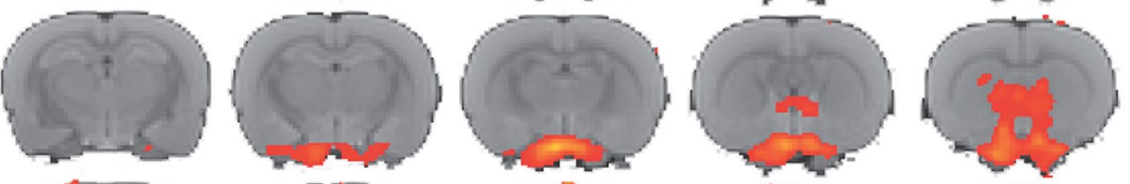

3
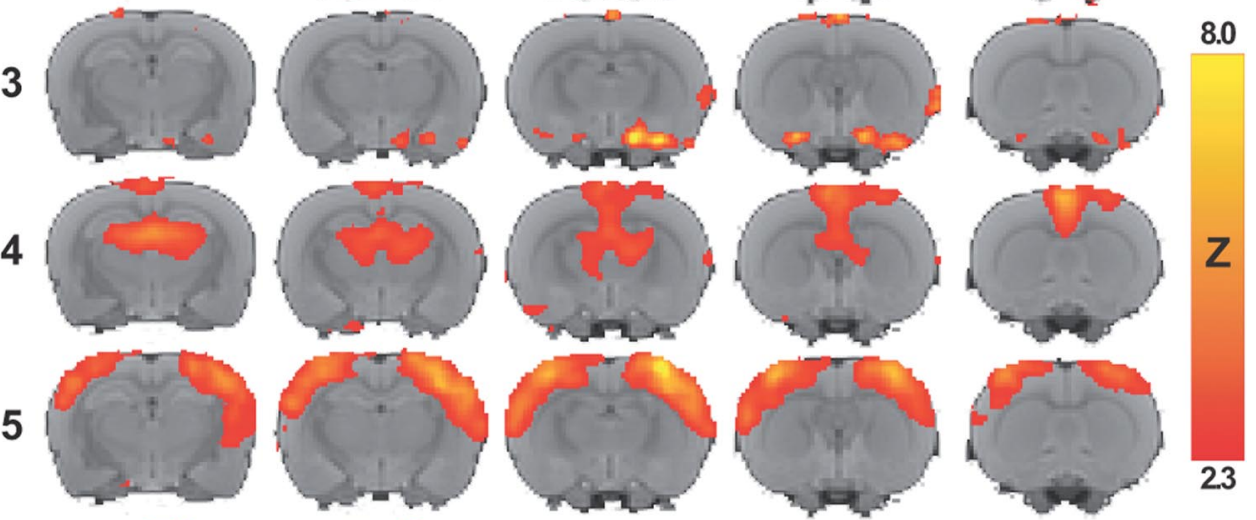

6
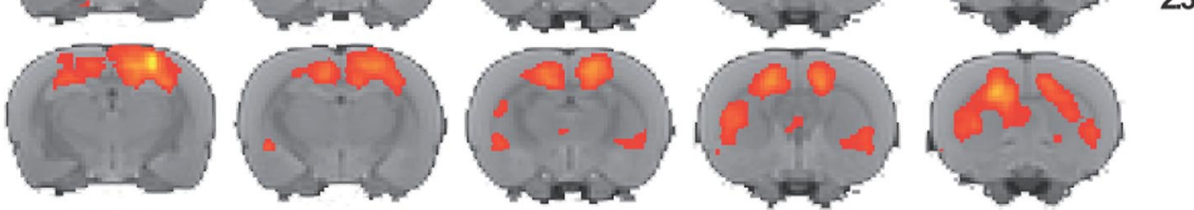

7
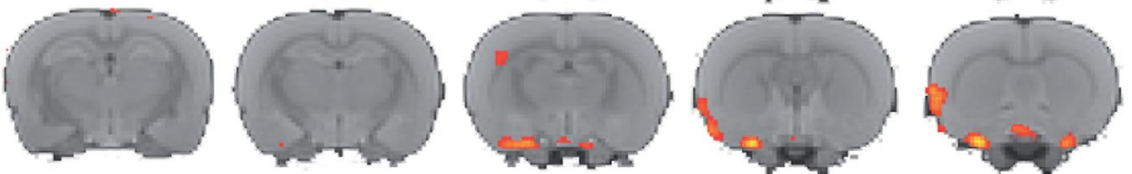

B
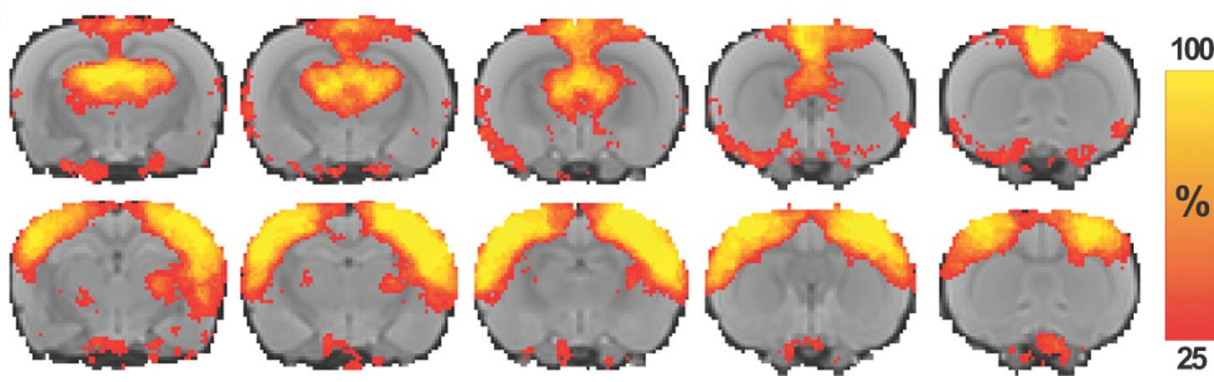

Figure 2. Functional connectivity clusters in rat brain. $A$, Maps of functional connectivity clusters as calculated by probabilistic independent component analysis of resting-state fMRI data from 20 healthy animals based on 7 components (color-coding reflects Z-scores). $\boldsymbol{B}$, Incidence of network components four (top; bilateral cingulate-thalamic network) and five (bottom; bilateral sensorimotor cortical network). Maps are overlaid on a multislice anatomical $\mathrm{T}_{2}$-weighted rat brain template.

after $>3$ weeks $(P<0.05$ vs dip at day 7$)$, while intraregional signal coherence in the ipsilesional sensorimotor cortex remained significantly lowered up to 10 weeks in the Stroke Large $_{\text {group }}(p<0.05$ vs baseline and controls). In the left, contralesional sensorimotor cor-

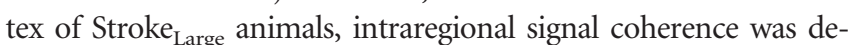
creased at 3 and $7 \mathrm{~d}$ poststroke ( $p<0.05$ vs baseline) followed by significant improvement at days 21 and 49 (Fig. 3C) as compared with the dip at day $7(p<0.05)$ (Fig. $4 C)$. In Stroke $_{\text {Medium }}$ animals no statistically significant changes occurred in intraregional signal coherence in the left, contralesional sensorimotor cortex.

The temporal changes in intercortical functional connectivity after stroke were confirmed by serial EEG recordings in a separate group of Stroke $\mathrm{Medium}_{\mathrm{m}}$ rats. In line with our resting-state fMRI findings, we measured a significant loss of intercortical synchro- nization of EEG signals in the low-frequency delta band $(1-5 \mathrm{~Hz})$ at day $3\left(z^{\prime}=0.69 \pm 0.12\right)$ as compared with baseline $\left(z^{\prime}=\right.$ $0.83 \pm 0.08 ; p<0.05)$, which significantly recovered at day 21 after stroke $\left(z^{\prime}=0.81 \pm 0.04 ; p<0.05\right.$ vs day 3$)$.

To compute changes in network characteristics in the bilateral sensorimotor cortices, we also performed graph analysis of the resting-state fMRI-based functional connectivity data. The normalized weighted clustering coefficient, $\gamma$, was increased subacutely after stroke in both stroke groups as compared with baseline and controls ( $p<0.05$ at days 3 and 7) (Fig. 4D), and declined thereafter. In Stroke ${ }_{\text {Medium }}$ animals, $\gamma$ had returned to baseline levels after $21 \mathrm{~d}$, but in the Stroke Large $_{\text {group it remained }}$ significantly elevated up to 10 weeks after stroke $(p<0.05$ vs baseline and controls). The normalized weighted shortest path 

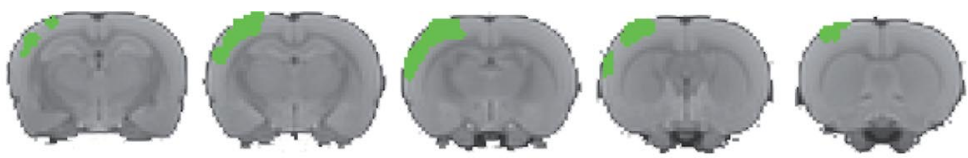

A

Stroke $_{\text {Medium }}$

B

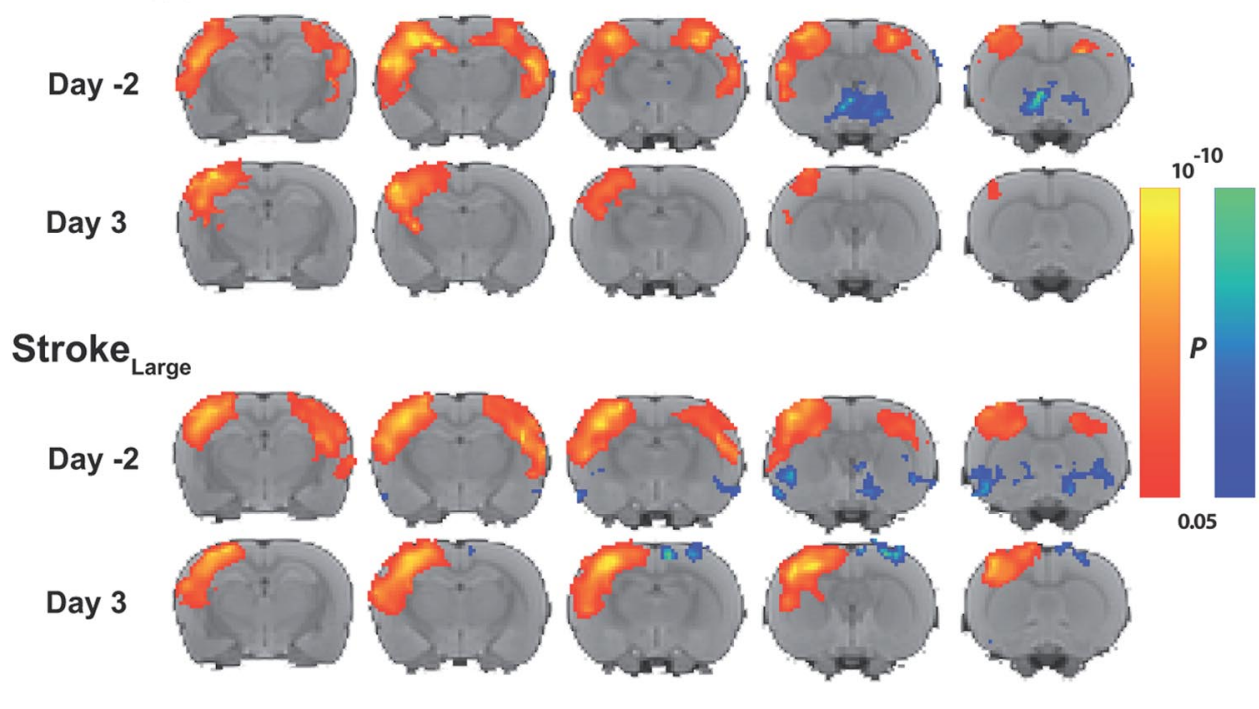

Stroke $_{\text {Medium }}$

Day 7
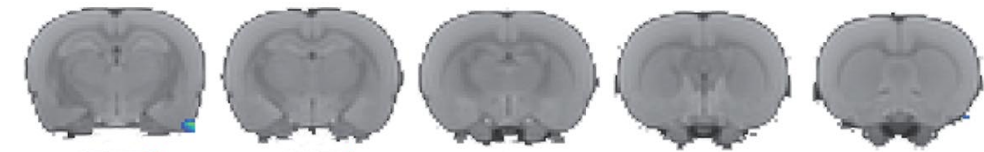

Day 21
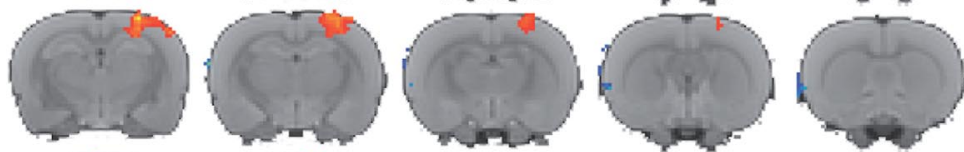

Day 49
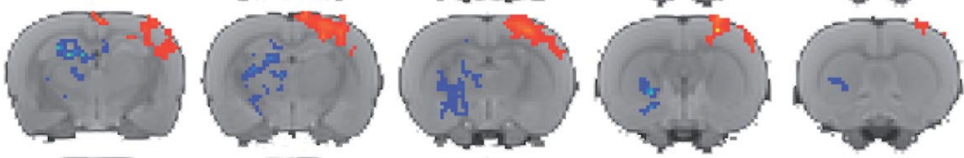

Day 70
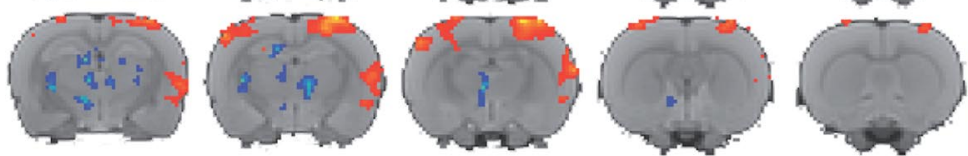

Stroke $_{\text {Large }}$

Day 7
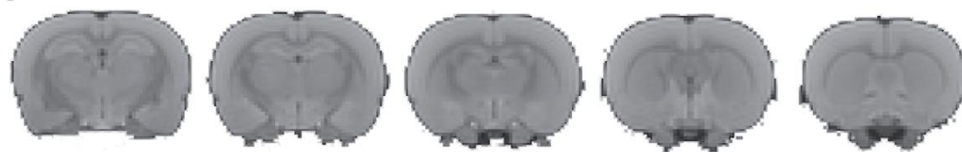

Day 21
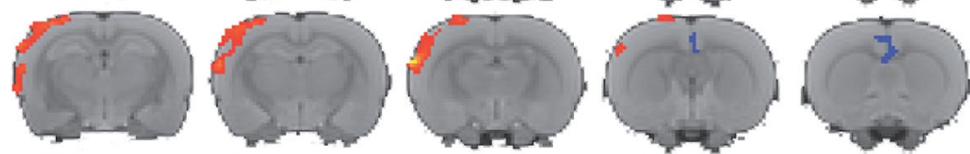

0.05

Day 49
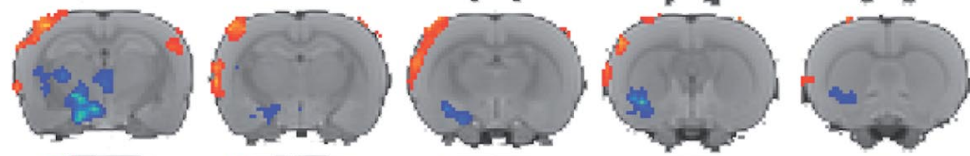

Day 70
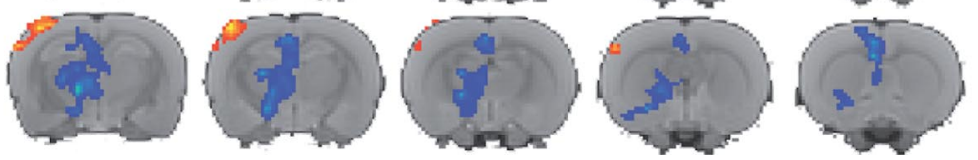

Figure 3. Functional connectivity with left (contralesional) sensorimotor cortex. $A$, Overlay of the left sensorimotor cortex ROl on consecutive coronal slices from a multislice anatomical $\mathrm{T}_{2}$-weighted rat brain template. This ROl was used as seed for functional connectivity analyses. $\boldsymbol{B}$, Group mean maps of functional connectivity ( $z^{\prime}$ ) with left sensorimotor cortex (Figure legend continues.) 

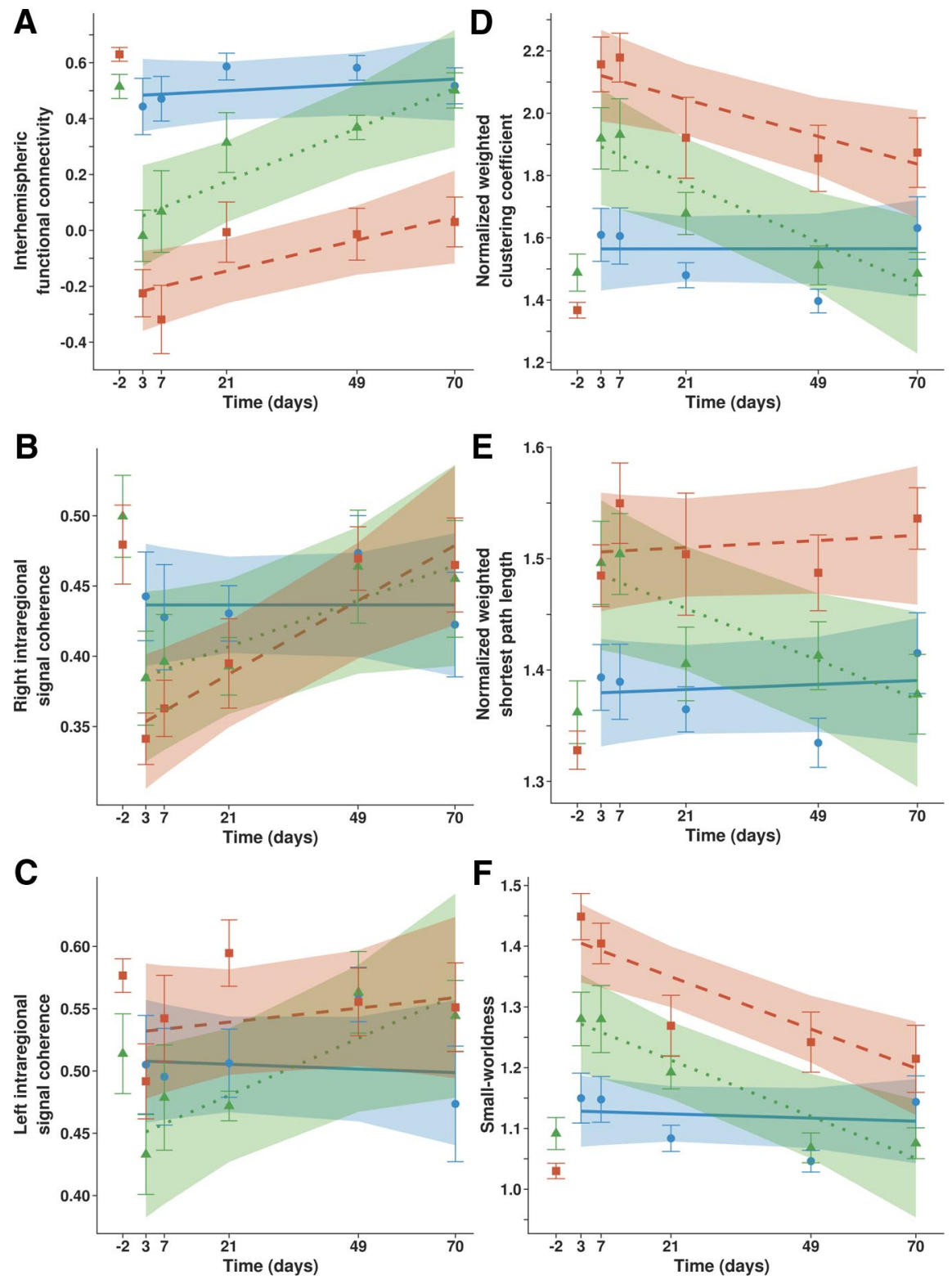

Figure 4. Interhemispheric functional connectivity and intraregional signal coherence of right (ipsilesional) and left (contralesional) sensorimotor cortices and network features of bilateral sensorimotor cortex. Mean values (symbols) \pm SEM (error bars), and linear mixed model estimated group patterns (lines), with 95\% confidence intervals (shading), of $(\boldsymbol{A})$ interhemispheric functional connectivity $\left(z^{\prime}\right)$ between left and right sensorimotor cortices; intraregional signal coherence $\left(z^{\prime}\right)$ of $(\boldsymbol{B})$ right (ipsilesional) and $(\boldsymbol{C})$ left (contralesional) sensorimotor cortices; and of $(\boldsymbol{D})$ normalized weighted clustering coefficient, $(\boldsymbol{E})$ normalized weighted shortest path length, and $(\boldsymbol{F})$ small-worldness in the bilateral sensorimotor cortical network, as a function of time in the Control (circles, solid lines; blue; $N=10$ ), Stroke ${ }_{\text {Medium }}$ (triangles, dotted lines; green; $N=5$ ), and Stroke Large $_{\text {(squares, dashed }}$ lines; red; $N=8$ ) groups. Baseline values at $2 \mathrm{~d}$ before stroke are only shown as mean \pm SEM.

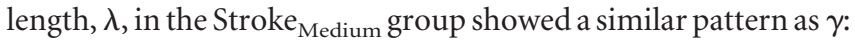
subacute rise ( $p<0.05$ vs baseline and controls at days 3 and 7$)$ which normalized after $21 \mathrm{~d}$ (Fig. 4E). In Stroke Large $\lambda$ was persistently increased over the entire poststroke time course of 10 weeks ( $p<0.05$ vs baseline and controls). Both stroke groups,

(Figure legend continued.) before and at $3 \mathrm{~d}$ poststroke in the Stroke ${ }_{\text {Medium }}$ and Stroke $\mathrm{Large}_{\text {a }}$ groups. C, Group mean maps of difference in functional connectivity with left sensorimotor cortex at days 7, 21, 49, and 70, as compared with day 3, in the Stroke Medium $_{\text {and Stroke }}$ Large groups. $P$ values range between 0.05 and $10^{-10}$ for both positive (red-yellow) and negative (blue-green) values.
Stroke $_{\text {Medium }}$ and Stroke $_{\text {Large, demon- }}$ strated significantly increased smallworldness, $\sigma(\gamma / \lambda)$, subacutely after stroke, as compared with baseline and controls ( $p<0.05$ at days 3 and 7) (Fig. $4 F)$. In Stroke Medium rats, $\sigma$ normalized after $21 \mathrm{~d}$. However, despite a gradual decline, $\sigma$ in Stroke $_{\text {Large }}$ remained significantly increased up to 7 weeks as compared with group Control $(p<0.05)$.

To validate these findings of longitudinal alterations in sensorimotor cortical network characteristics after stroke, we performed the same network analysis on serial resting-state fMRI data from our previous study. Similar to the current study, we found that $\gamma$ and $\sigma$ significantly declined from day $3(\gamma=1.52 \pm 0.19$, subcortical injury and $1.75 \pm 0.29$, subcortical and cortical injury and $\sigma=$ $1.05 \pm 0.08$, subcortical injury and $1.17 \pm$ 0.13 , subcortical and cortical injury) to day 70 after stroke $(\gamma=1.43 \pm 0.16$, subcortical injury and $1.46 \pm 0.15$, subcortical and cortical injury and $\sigma=1.04 \pm 0.07$, subcortical injury and $1.06 \pm 0.09$ subcortical and cortical injury; $p<0.05$ ). $\lambda$ also decreased between subacutely and chronically in both stroke groups from our previous study, similar to Stroke Medium. $_{\text {. }}$.

\section{Structural integrity of white matter tracts}

In addition to measurement of signal coherence and functional connectivity, MRI was used to assess structural changes in white matter regions that encompass key connections of the sensorimotor network. We applied tract-based spatial statistics on FA data to depict significant differences in FA in whole-brain white matter between measurements before and $3 \mathrm{~d}$ after stroke (Fig. 5A). The Stroke ${ }_{\text {Medium }}$ and Stroke $_{\text {Large }}$ groups displayed FA reductions in the internal and external capsules inside and nearby the lesion $(p<0.05$ vs baseline and controls (Fig. 6C,D). In addition, FA was reduced in the ipsilesional cerebral peduncle in both stroke groups.

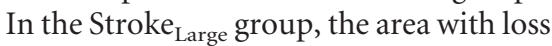
of FA was more extensive and included the corpus callosum and white matter tracts in the contralesional hemisphere. Indications for increases in FA at $3 \mathrm{~d}$ after stroke were found in some contralesional white matter areas in both groups and near the midline in Stroke $_{\text {Large. }}$. These findings should be interpreted with caution, as such changes may be related to midline shifts that occur around this stage after a large stroke.

Control animals demonstrated significant FA increase in all white matter ROIs, as demonstrated in Figure $5 B$, which displays maps of changes in FA between the early $3 \mathrm{~d}$ and chronic $70 \mathrm{~d}$ time points based on tract-based spatial statistics and in Figure $6 A-D$, which shows time courses of FA in corpus callosum, right cerebral peduncle, and the right anterior and posterior internal 


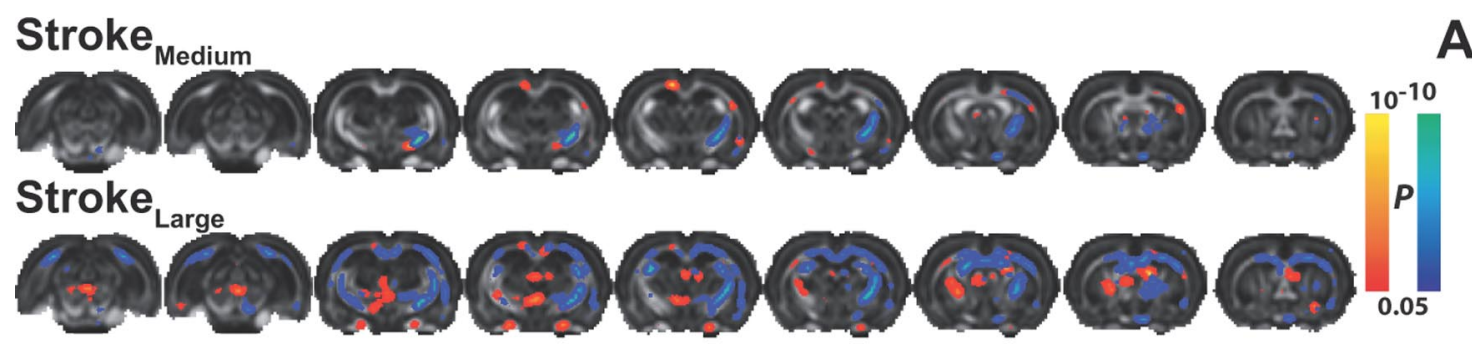

Control

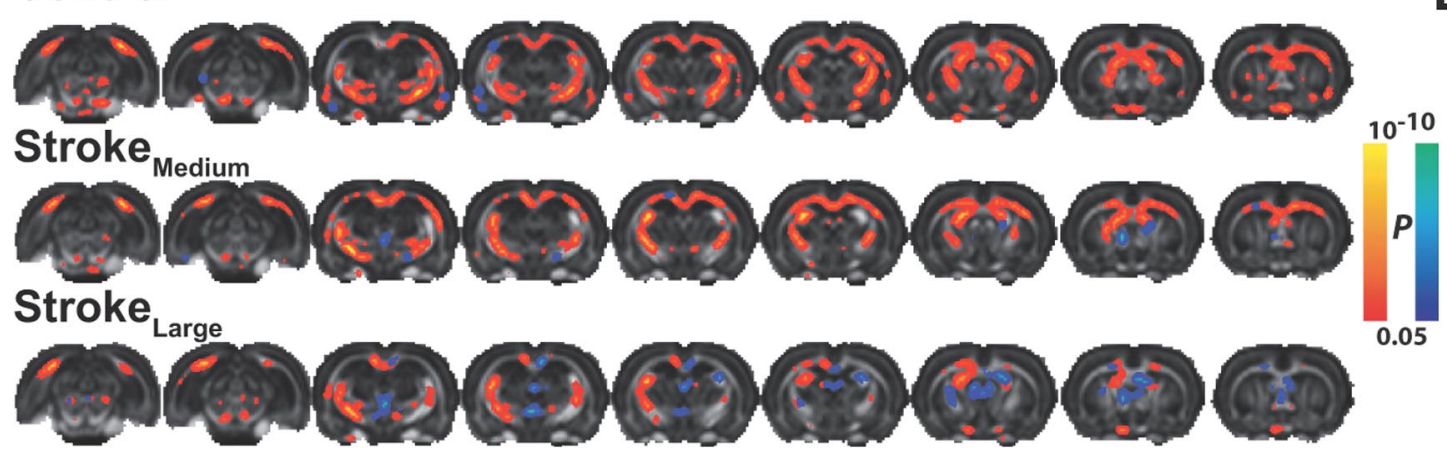

B

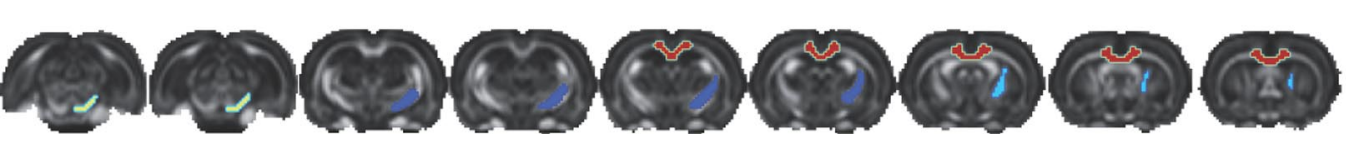

C

Figure 5. FA changes in white matter tracts. Maps of differences in white matter FA (overlaid on consecutive coronal slices from a multislice FA rat brain template) (A) between prestroke and $3 \mathrm{~d}$

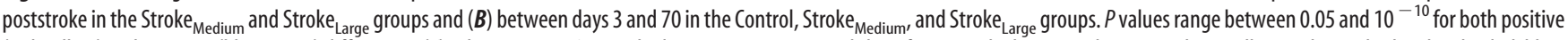
(red-yellow) and negative (blue-green) differences. (C) White matter ROIs overlaid on consecutive coronal slices from a multislice FA rat brain template. Yellow, right cerebral peduncle; dark blue, right posterior internal capsule; light blue, right anterior internal capsule; and red, corpus callosum.

capsules (ROIs outlined in Fig. $5 C)(p<0.05)$. A similar pattern was observed in the Stroke $_{\text {Medium }}$ group, except for white matter tracts inside the lesion area. After 49 and $70 \mathrm{~d}$, FA in the ipsilesional corticospinal tract of Stroke $_{\text {Medium }}$ rats was not significantly reduced or even elevated (anterior internal capsule $(p<$ 0.05 ) as compared with control rats (Fig. 6). Significant improvement from its baseline value at day 70 in the ipsilesional cerebral peduncle (Fig. $6 B)$ and anterior internal capsule (Fig. 6C) $(p<$ $0.05)$ was observed in Stroke Large $_{\text {animals. Significant group } \times}$ time effects for the FA changes in these ROIs in both stroke groups denoted a faster progression of FA as compared with controls $(p<0.05)$. In other ipsilesional white matter areas, the developmental rise in FA was largely diminished in Stroke $_{\text {Large }}$ animals, and significant decreases were even found in parts of the corpus callosum (Fig. 6A) ( $p<0.05$ vs baseline and controls). FA in all ipsilesional corticospinal tract ROIs of Stroke Large $_{\text {rats re- }}$ mained significantly lower than in the control group $(p<0.05)$. Contralesional (left) corticospinal tract regions still showed significant FA increase during the period of 10 weeks (Fig. 5B) and we found no significant differences in FA time courses between stroke and control groups.

Data from Luxol Fast Blue staining confirmed our DTI findings. Figure 7 demonstrates that high FA in the anterior part of the ipsilesional anterior capsule just outside the infarction (evident on the $\mathrm{H} \& \mathrm{E}$-stained section) in a Stroke Large $_{\text {animal corresponded with }}$ high levels of myelin. Furthermore, correlation analysis of normalized in vivo FA versus normalized postmortem Luxol Fast Blue staining intensity in various left and right white matter regions (i.e., corpus callosum, internal capsule, and cerebral peduncle) of rats from groups Control $(N=3)$ and Stroke Large $(N=3)$, revealed positive correlation in the control $(r=0.64 ; p<0.05)$ and stroke group $(r=0.32)$.

Correlations between changes in functional connectivity and white matter integrity

Correlation between interhemispheric functional and structural connectivity as measured with resting-state fMRI and DTI, respectively, was tested by calculating the correlation coefficient between intercortical functional connectivity (between left and right sensorimotor cortices) and FA (in the corpus callosum). A significant positive relationship was evident for all groups and time points pooled together $(r=0.38 ; p<0.05)$.

To evaluate whether shifts in intercortical functional connectivity are associated with alterations in white matter integrity after stroke, we correlated changes in functional connectivity with changes in FA (in corpus callosum and ipsilesional corticospinal tract regions) between consecutive poststroke time points. Results are shown in Ta-

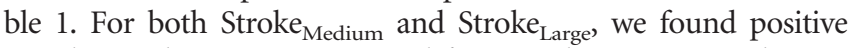
correlations between intercortical functional connectivity changes and changes in FA for all ROIs in the ipsilesional corticospinal tract, but not between functional connectivity changes and changes in FA in the contralesional corticospinal tract.

\section{Correlations between functional recovery and brain reorganization}

Correlation coefficients between recovery of sensorimotor function (changes in sensorimotor performance score between consecutive poststroke time points) and functional indices of reorganization in the bilateral sensorimotor cortices (changes in interhemispheric functional connectivity, intraregional signal 


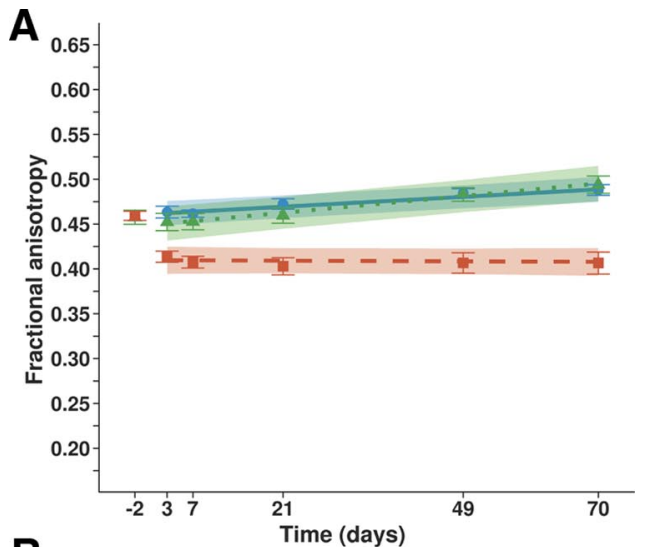

B
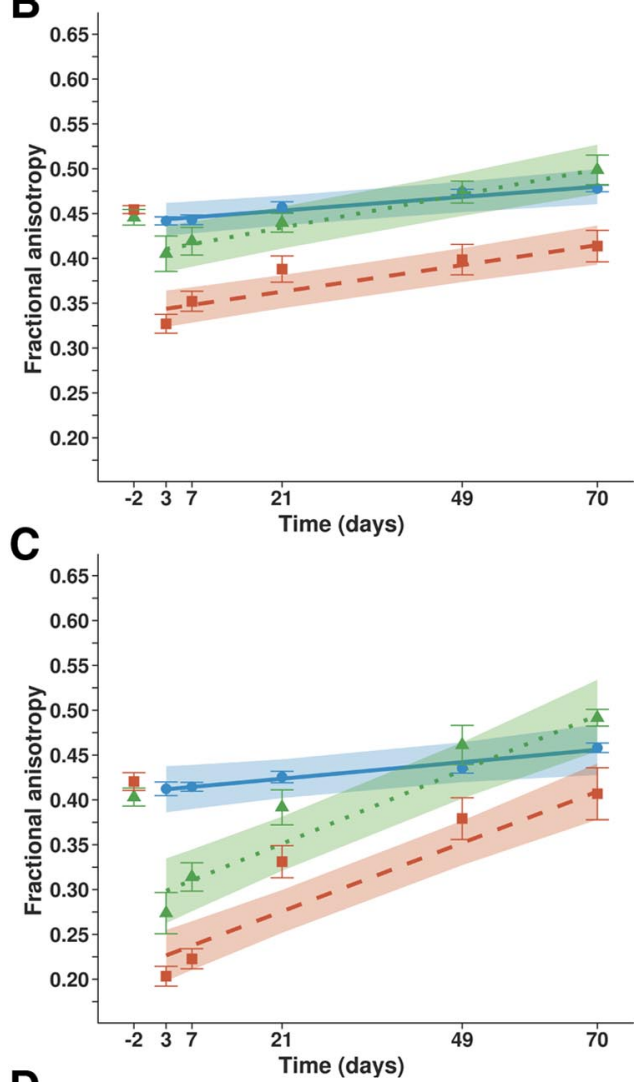

D

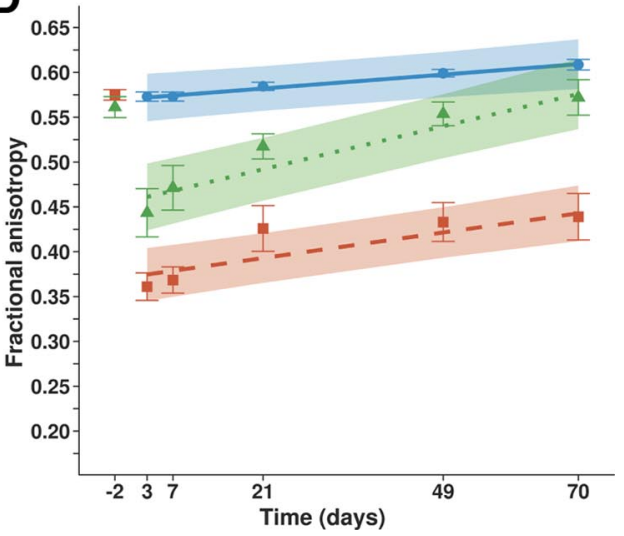

Figure 6. FA in white matter ROls. Mean values (symbols) \pm SEM (error bars), and linear mixed model estimated group patterns (lines), with 95\% confidence intervals (shading), of FA in ( $A$ ) corpus callosum, $(\boldsymbol{B})$ right (ipsilesional) cerebral peduncle, $(\boldsymbol{C})$ right (ipsilesional) anterior internal capsule, and (D) right (ipsilesional) posterior internal capsule, as a function of time in the Control (circles, solid

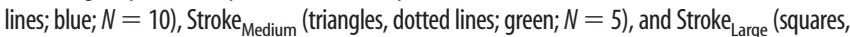
dashed lines; red; $N=8$ ) groups. Baseline values at 2 d before stroke are only shown as mean \pm SEM. coherence, network clustering coefficient, network shortest path length, and network small-worldness, respectively, between consecutive poststroke time points) are shown in Table 2. We combined data from our current and our previous study, in which we also serially conducted sensorimotor function testing and resting-state fMRI in rats with different stroke lesion sizes (van Meer et al., 2010b). We first tested for significant differences between the two studies, by adding "study" as fixed effect in our linear mixed model analysis, and found study to be nonsignificant for all variables. Both medium and large stroke groups revealed highest significant correlation coefficients between behavioral recovery and restoration of interhemispheric functional connectivity. On the other hand, sensorimotor function changes correlated negatively with changes in bilateral sensorimotor cortex network parameters, i.e., clustering coefficient, shortest path length, and small-worldness, which was statistically significant in animals with a large stroke. We found no consistent correlation between changes in sensorimotor performance and changes in signal coherence in right or left sensorimotor cortex for the stroke groups. Also, no significant correlations were found between changes in sensorimotor function and changes in functional network measures for the bilateral cingulate-thalamic network (data not shown).

Table 3 shows the correlation coefficients of changes in sensorimotor performance scores versus changes in DTI-derived FA in corpus callosum, the anterior and posterior parts of the right (ipsilesional) internal capsule, and the right (ipsilesional) cerebral peduncle, as calculated from changes between all consecutive time points. Significant correlation coefficients were observed in the Stroke $_{\text {Large }}$ group for all white matter ROIs and only for the ipsilesional internal capsule in the $S_{\text {troke }}{ }_{\text {Medium }}$ group.

\section{Relationship between early brain reorganization and chronic functional outcome}

To evaluate whether chronic functional outcome is related to early MRI indices of functional or structural connectivity, we measured the correlation coefficient between sensorimotor performance score at day 70 versus interhemispheric functional connectivity, intraregional signal coherence, network parameters (in sensorimotor cortices), and FA (in corpus callosum and ipsilesional corticospinal tract regions) at day 3 after stroke for all

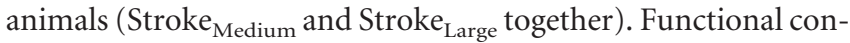
nectivity, signal coherence, and network parameters at $3 \mathrm{~d}$ poststroke all had absolute correlation coefficients with final functional outcome that were lower than 0.33 . However, FA in the corpus callosum and ipsilesional corticospinal tract ROIs at $3 \mathrm{~d}$ poststroke demonstrated significant correlation with sensorimotor performance score after $70 \mathrm{~d}(\rho=0.53,0.77,0.74$, and 0.80 for corpus callosum, anterior internal capsule, posterior internal capsule, and cerebral peduncle, respectively; $p<0.05$ ). Notably, the correlations of final sensorimotor performance score with subacute FA in the ipsilesional corticospinal tract were of the same order as correlations with the initial sensorimotor performance score $(\rho=0.67 ; p<0.05)$ or lesion volume ( $\rho=$ $-0.72 ; p<0.05)$ at $3 \mathrm{~d}$ poststroke.

\section{Discussion}

We applied serial resting-state fMRI and DTI in combination with advanced network analysis methods to elucidate the interaction between changes in gray matter functional connectivity, white matter structural integrity, and network topology of the bilateral sensorimotor system in relation to functional recovery after medium and large unilateral stroke in rats. Our main find- 

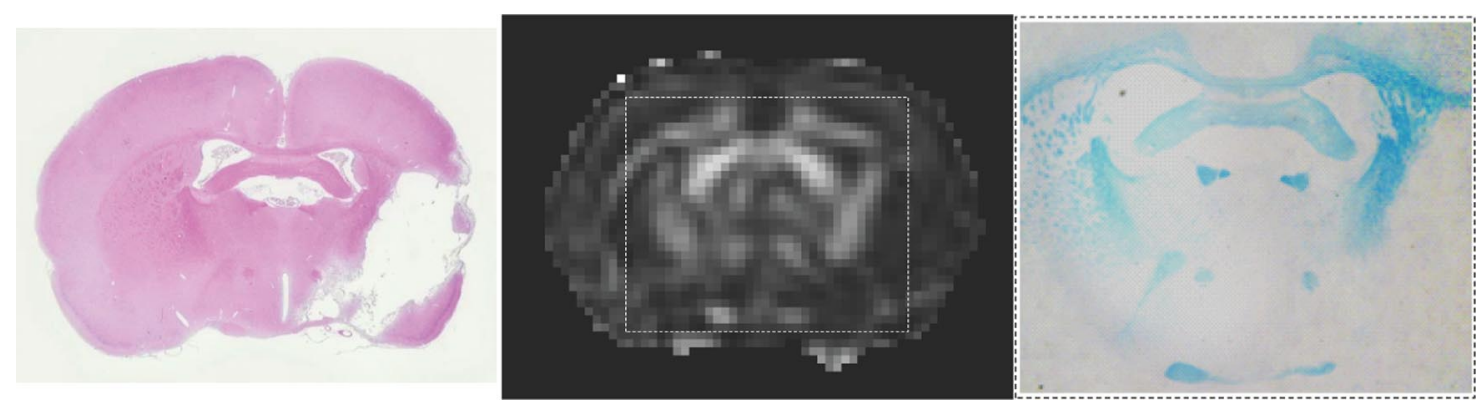

Figure 7. FA and Luxol Fast Blue staining in perilesional white matter. H\&E-stained section (left), FA map (middle), and Luxol Fast Blue-stained section (right) (corresponding with dashed rectangle in $F A$ map) from a coronal rat brain slice at $70 \mathrm{~d}$ after a large stroke.

Table 1. Correlations of changes in interhemispheric functional connectivity (between right (ipsilesional) and left (contralesional) sensorimotor cortex) versus changes in fractional anisotropy in corpus callosum and right corticospinal tract regions

\begin{tabular}{lllll}
\hline & $\begin{array}{l}\text { Corpus } \\
\text { callosum }\end{array}$ & $\begin{array}{l}\text { Internal capsule, } \\
\text { anterior }\end{array}$ & $\begin{array}{l}\text { Internal capsule, } \\
\text { posterior }\end{array}$ & $\begin{array}{l}\text { Cerebral } \\
\text { peduncle }\end{array}$ \\
\hline Stroke $_{\text {Medium }}$ & 0.14 & $0.70^{*}$ & $0.84^{*}$ & $0.58^{*}$ \\
Stroke $_{\text {Large }}$ & 0.33 & $0.72^{*}$ & $0.69^{*}$ & $0.63^{*}$
\end{tabular}

Correlation coefficients calculated from linear mixed model analysis of changes in interhemispheric functional connectivity (between left and right sensorimotor cortex) versus changes in fractional anisotropy in corpus callosum, right (ipsilesional) anterior and posterior internal capsule, and right (ipsilesional) cerebral peduncle, between successive time points in the Stroke Medium $_{\text {and Stroke }}$ Large groups. ${ }^{*} P<0.05$.

ings were that (1) improvement of sensorimotor function correlates with restoration of interhemispheric functional connectivity and normalization of network configuration of the bilateral sensorimotor cortex, (2) gradual recovery of sensorimotor function after a large stroke correlates with increased structural integrity of the ipsilesional corticospinal tract, (3) restoration of interhemispheric functional connectivity between bilateral sensorimotor cortices is associated with unilateral improvement of structural integrity of the ipsilesional corticospinal tract, and (4) good functional outcome is linked with preserved or repaired structural integrity within the ipsilesional corticospinal tract.

The functioning of brain networks relies on synchronization of intrinsic neuronal signaling, reflective of functional connectivity, which dynamically alters within and between hemispheres after experimental unilateral stroke. Our findings of early loss followed by recovery of interhemispheric functional connectivity are in line with pioneering studies in patients (He et al., 2007) and animal models (van Meer et al., 2010b) that have explored the impact of acute ischemic injury and subsequent recovery on resting-state fMRI signals. Although in animal studies anesthesia may affect functional connectivity measurements, we and others have shown that coherence of low-frequency BOLD signal fluctuations between bilateral homologous sensorimotor regions is preserved at $1 \%$ isoflurane (Wang et al., 2011) and correlates with slow power modulations of local field potentials in rats (Pan et al., 2011). We here confirm the neurophysiological basis of strokeinduced functional connectivity changes by showing parallel shifts in the correlation coefficient of interhemispheric lowfrequency BOLD signals and interhemispheric low-frequency delta EEG signals. Furthermore, we successfully applied a modelfree probabilistic independent component analysis strategy to identify a group-based functional bilateral sensorimotor cortical network in rat brain, in addition to other networks such as a bilateral cingulate-thalamic network. Despite differences in rat strain, anesthesia, ICA algorithm, and number of components, our findings are largely in agreement with previous studies that also demonstrated predominantly bilateral functional networks in rat brain (Hutchison et al., 2010; Jonckers et al., 2011). Subsequent analyses demonstrated that both interhemispheric functional connectivity and ipsilesional intrahemispheric signal coherence in sensorimotor cortex and cingulate-thalamic network were significantly reduced after medium and large stroke, particularly at subacute stages. Concurrently, different network features, viz. the clustering coefficient (measure of functional segregation within networks), shortest path length (measure of functional integration within networks), and small-worldness (measure of functional network efficiency), were significantly increased within the bilateral sensorimotor cortex. These findings were confirmed by retrospective graph-based network analyses of the resting-state fMRI data from our previous study (van Meer et al., 2010b), which also revealed high initial values of these network parameters before chronic decline. We speculate that the increase in small-worldness in the bilateral sensorimotor cortical network may be related to early stroke-induced plasticity mechanisms. For instance, initial recruitment or unmasking of diffuse redundant neuronal pathways, or reset of synaptic activity, may lead to loss of synchronization of signaling (with reduced cortical signal coherence and functional connectivity) while increasing random integration between neurons, creating a state of overconnectivity (with increased network clustering and shortest path length). This might be associated with spine elongation (Brown et al., 2008), neurite sprouting (Stroemer et al., 1995), patterned spontaneous neuronal activity (Carmichael and Chesselet, 2002), and a state of extensive hyperexcitability (Fujioka et al., 2004), which have been observed during initial phases of poststroke recovery. At later stages dendritic pruning (Jones and Schallert, 1992) and synaptogenesis (Stroemer et al., 1995) occur, which institutes selective neuronal connections while others are abolished, leading to refinement of the reorganized network and stabilization of functioning (Murphy and Corbett, 2009). The establishment and maturation of neuronal circuitry, with diminishment of superfluous neuronal clustering and wiring, may bring about the restoration of ipsilesional intracortical signal coherence and intercortical functional connectivity, and normalization of small-world network topology, i.e., optimal network configuration for global information transfer and local processing, that we observed chronically. However, other factors such as alterations in hemodynamics and neurovascular coupling may also significantly contribute to the observed changes. Contrary to

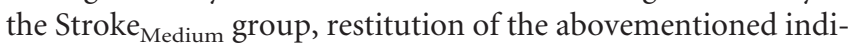
ces of brain functionality was incomplete in Stroke Large $_{\text {rats }}$ and accompanied by a significant increase in signal coherence within the contralesional sensorimotor cortex, which may be associated with enhanced neuroanatomical connectivity (van Meer et al., 
Table 2. Correlations of changes in behavioral function versus changes in functional network measures

\begin{tabular}{|c|c|c|c|c|c|c|}
\hline & \multirow[b]{2}{*}{ Functional connectivity } & \multicolumn{2}{|l|}{ Signal coherence } & \multirow[b]{2}{*}{ Clustering coefficient } & \multirow{2}{*}{$\begin{array}{l}\text { Shortest path } \\
\text { length }\end{array}$} & \multirow[b]{2}{*}{ Small-worldness } \\
\hline & & Right & Left & & & \\
\hline Stroke $_{\text {Medium }}$ & $0.40^{*}$ & 0.14 & 0.18 & -0.13 & -0.19 & -0.12 \\
\hline [all] & $(0.12-0.69)$ & $(-0.17-0.45)$ & $(-0.13-0.49)$ & $(-0.44-0.18)$ & $(-0.50-0.12)$ & $(-0.43-0.18)$ \\
\hline Stroke $_{\text {Large }}$ & $0.50^{*}$ & 0.25 & 0.16 & $-0.48^{*}$ & $-0.42^{*}$ & $-0.40^{*}$ \\
\hline [all] & $(0.29-0.72)$ & $(-0.01-0.52)$ & $(-0.11-0.43)$ & $(-0.71--0.24)$ & $(-0.65-0.19)$ & $(-0.66--0.15)$ \\
\hline
\end{tabular}

Correlation coefficients (and 95\% confidence intervals) calculated from linear mixed model analysis of ranked changes in sensorimotor performance score versus changes in (1) functional connectivity between right and left sensorimotor cortices, (2) signal coherence in right and left sensorimotor cortices, (3) normalized weighted clustering coefficient in bilateral sensorimotor cortex, (4) normalized weighted shortest path length in bilateral sensorimotor cortex, and (5)

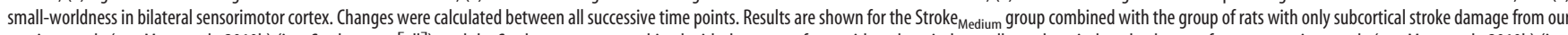

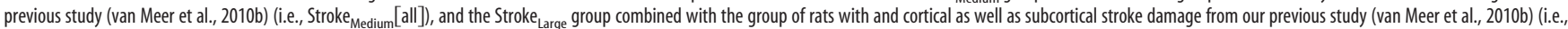
Stroke $_{\text {Large }}[$ all $\left.]\right) .{ }^{*} P<0.05$.

Table 3. Correlations of changes in behavioral function versus changes in white matter integrity

\begin{tabular}{lllll}
\hline & $\begin{array}{l}\text { Corpus } \\
\text { callosum }\end{array}$ & $\begin{array}{l}\text { Internal capsule, } \\
\text { anterior }\end{array}$ & $\begin{array}{l}\text { Internal capsule, } \\
\text { posterior }\end{array}$ & $\begin{array}{l}\text { Cerebral } \\
\text { peduncle }\end{array}$ \\
\hline Stroke $_{\text {Medium }}$ & -0.16 & $0.39^{*}$ & 0.22 & 0.22 \\
Stroke $_{\text {Large }}$ & $0.61^{*}$ & $0.70^{*}$ & $0.80^{*}$ & $0.57^{*}$ \\
\hline
\end{tabular}

Correlation coefficients calculated from linear mixed model analysis of ranked changes in sensorimotor performance score versus changes in fractional anisotropy in corpus callosum, right (ipsilesional) anterior and posterior internal capsule, and right (ipsilesional) cerebral peduncle, between successive time points in the Stroke Medium $_{\text {and Stroke- }}$ Large groups. ${ }^{*} P<0.05$.

2010a). The recruitment of contralesional regions may play an important supportive or compensatory role in functional recovery after extensive unilateral damage to functional networks.

Our data on changes in network features of the sensorimotor cortex are in contrast with findings by Wang et al. (2010), who observed a decrease in clustering coefficient and a permanent shift toward a random network configuration in the motor system at one year after subcortical stroke in human subjects, which we associated with random spontaneous axonal outgrowth. This dissimilarity may be explained by differences between the (re)organization of rat and human brain, but may also be related to differences in the analytical method. Instead of an ROI-based approach, we applied voxel-based graph analysis, as voxel-based networks exhibit properties of a small-world network more prominently and are more robust against network fragmentation (Hayasaka and Laurienti, 2010).

Our data show that modifications in gray matter organization of the bilateral sensorimotor network were accompanied by changes in white matter integrity. Significant reductions in FA, which was histologically associated with loss of myelin staining, were observed in key white matter tracts. Particularly after large stroke, these changes were not limited to the lesioned area but extended to perilesional and contralesional parts, e.g., the corpus callosum, cerebral peduncles, and contralesional corticospinal tract, indicative of anterograde (Wallerian) or retrograde degeneration or widespread edema. Nonetheless, a subsequent steady rise in FA in most white matter areas, similar to control animals and corresponding with ongoing maturation during early adulthood-the developmental stage of the rats in our study (Lebel et al., 2010; Westlye et al., 2010)—suggested reinstatement of normal white matter development, possibly accompanied by edema resolution and additional structural remodeling. Our rat data are in accordance with findings by Schaechter et al. (2009), who detected FA elevations in the ipsilesional and contralesional corticospinal tract of chronic stroke patients. Furthermore, significantly higher FA values in perilesional white matter, as we observed in the anterior internal capsule of Stroke Medium $_{\text {rats, has }}$ been histologically associated with increased axonal density and myelination in a similar stroke model (Jiang et al., 2006). How- ever, other factors such as gliosis may also contribute (van der Zijden et al., 2008b).

Our study reveals a significant correlation between intercortical synchronization of resting-state fMRI signals and FA in the corpus callosum in control and stroke groups, which reflects the link between (interhemispheric) functional and structural connectivity. Restoration of functional connectivity between left and right sensorimotor cortices after stroke correlated most strongly with recovery of FA in the ipsilesional internal capsule, particularly after a large stroke. This underscores the critical role of intact unilateral corticospinal pathways for interhemispheric signal synchronization, which, we believe, institutes an efficient balance of bilateral somatosensory input and motor output signals. Whether reinstatement of intercortical coherence of neuronal activity purely relies on, or possibly also contributes to, recovery of ipsilesional corticospinal tract integrity remains unclear.

An important question is to what degree distinct modifications in neuronal networks contribute to functional improvement after stroke. In subacute human stroke subjects it has been shown that upper extremity impairment correlates with disruption of interhemispheric functional connectivity in the somatomotor network and degree of corticospinal tract damage (Carter et al., 2010, 2012). Analysis of our current and previous restingstate fMRI data in rats demonstrated that incremental changes in sensorimotor function between poststroke time points particularly correlates with increases in interhemispheric functional connectivity between the ipsilesional and contralesional sensorimotor cortices after medium and large stroke, and with decreases of clustering coefficient, shortest path length, and smallworldness of the bilateral sensorimotor cortical network after

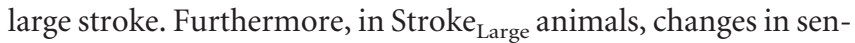
sorimotor performance scores were highly correlated with changes in FA in corpus callosum, ipsilesional anterior and posterior internal capsule, and ipsilesional cerebral peduncle. This points toward a critical dependence of functional recovery on large-scale functional and structural brain reorganization after large stroke. Large stroke lesions with damage to somatosensory or motor cortex and subcortical ascending and descending white matter tracts largely disrupt signaling within and consequent functioning of the sensorimotor network, in line with findings in human stroke subjects (Carter et al., 2012). Functional recovery would therefore considerably rely on recruitment of contralesional homologous cortical tissue and significant rewiring of the corticospinal tracts, leading to large shifts in network configuration. The temporary network disturbance after medium stroke, with only limited or no damage to the somatosensory cortex and minor injury to the corticospinal tract, may resolve within relatively short time, without the necessity of extensive long-lasting modifications in network configuration, leading to more rapid functional recovery. It should be mentioned though that more 
specific behavioral tests may reveal more subtle differences and shifts in sensorimotor function, as well as unidentified correlations with changes in neuronal network connectivity, particularly after medium or small stroke.

Establishing whether measurable early alterations in structural or functional network organization inform on final outcome would be of critical importance for early diagnosis, prediction, and treatment selection. In our study functional outcome after 10 weeks was not related to one of the MRI-based measures of sensorimotor cortical functional connectivity or network characteristics at $3 \mathrm{~d}$ poststroke. However, final sensorimotor performance outcome correlated positively with FA in ipsilesional corticospinal tract regions subacutely, where poor functional outcome was linked to subacutely low FA, in line with DTI studies in human patients (Thomalla et al., 2004; Puig et al., 2010; Radlinska et al., 2010). Furthermore, a recent experimental rehabilitation trial demonstrated that greater interventioninduced gains in motor function were related to higher FA in transcallosal and ipsilesional corticospinal tracts of chronic stroke patients (Lindenberg et al., 2012). Thus, preserved structural integrity of the ipsilesional corticospinal tract appears to be a critical factor for good functional outcome after unilateral stroke and could therefore be a primary target for therapeutic repair strategies.

\section{References}

Basser PJ, Pierpaoli C (1996) Microstructural and physiological features of tissues elucidated by quantitative-diffusion-tensor MRI. J Magn Reson B 111:209-219.

Beckmann CF, DeLuca M, Devlin JT, Smith SM (2005) Investigations into resting-state connectivity using independent component analysis. Philos Trans R Soc Lond B Biol Sci 360:1001-1013.

Brown CE, Wong C, Murphy TH (2008) Rapid morphologic plasticity of peri-infarct dendritic spines after focal ischemic stroke. Stroke 39:1286-1291.

Calautti C, Baron JC (2003) Functional neuroimaging studies of motor recovery after stroke in adults: a review. Stroke 34:1553-1566.

Carmichael ST (2003) Plasticity of cortical projections after stroke. Neuroscientist 9:64-75.

Carmichael ST, Chesselet MF (2002) Synchronous neuronal activity is a signal for axonal sprouting after cortical lesions in the adult. J Neurosci 22:6062-6070.

Carter AR, Astafiev SV, Lang CE, Connor LT, Rengachary J, Strube MJ, Pope DL, Shulman GL, Corbetta M (2010) Resting interhemispheric functional magnetic resonance imaging connectivity predicts performance after stroke. Ann Neurol 67:365-375.

Carter AR, Patel KR, Astafiev SV, Snyder AZ, Rengachary J, Strube MJ, Pope A, Shimony JS, Lang CE, Shulman GL, Corbetta M (2012) Upstream dysfunction of somatomotor functional connectivity after corticospinal damage in stroke. Neurorehabil Neural Repair 26:7-19.

Cox RW (1996) AFNI: software for analysis and visualization of functional magnetic resonance neuroimages. Comput Biomed Res 29:162-173.

Fagiolo G (2007) Clustering in complex directed networks. Phys Rev E Stat Nonlin Soft Matter Phys 76:026107.

Fox MD, Zhang D, Snyder AZ, Raichle ME (2009) The global signal and observed anticorrelated resting state brain networks. J Neurophysiol 101:3270-3283.

Fujioka H, Kaneko H, Suzuki SS, Mabuchi K (2004) Hyperexcitabilityassociated rapid plasticity after a focal cerebral ischemia. Stroke 35:e346-8.

Hayasaka S, Laurienti PJ (2010) Comparison of characteristics between region- and voxel-based network analyses in resting-state fMRI data. Neuroimage 50:499-508.

He BJ, Snyder AZ, Vincent JL, Epstein A, Shulman GL, Corbetta M (2007) Breakdown of functional connectivity in frontoparietal networks underlies behavioral deficits in spatial neglect. Neuron 53:905-918.

Humphries MD, Gurney K (2008) Network 'small-world-ness': a quantitative method for determining canonical network equivalence. PLoS One 3:e002051.
Hutchison RM, Mirsattari SM, Jones CK, Gati JS, Leung LS (2010) Functional networks in the anesthetized rat brain revealed by independent component analysis of resting-state FMRI. J Neurophysiol 103:3398-3406.

Ihaka R, Gentleman R (1996) R: A language for data analysis and graphics. J Comput Graph Stat 5:299-314.

Jiang Q, Zhang ZG, Ding GL, Silver B, Zhang L, Meng H, Lu M, PourabdillahNejed-D S, Wang L, Savant-Bhonsale S, Li L, Bagher-Ebadian H, Hu J, Arbab AS, Vanguri P, Ewing JR, Ledbetter KA, Chopp M (2006) MRI detects white matter reorganization after neural progenitor cell treatment of stroke. Neuroimage 32:1080-1089.

Jonckers E, Van Audekerke J, De Visscher G, Van der Linden A, Verhoye M (2011) Functional connectivity fMRI of the rodent brain: comparison of functional connectivity networks in rat and mouse. PLoS One 6:e18876.

Jones TA, Schallert T (1992) Overgrowth and pruning of dendrites in adult rats recovering from neocortical damage. Brain Res 581:156-160.

Klein S, Staring M, Murphy K, Viergever MA, Pluim JP (2010) Elastix: a toolbox for intensity-based medical image registration. IEEE Trans Med Imaging 29:196-205.

Laird NM, Ware JH (1982) Random-effects models for longitudinal data. Biometrics 38:963-974

Latora V, Marchiori M (2001) Efficient behavior of small-world networks. Phys Rev Lett 87:198701.

Lebel C, Caverhill-Godkewitsch S, Beaulieu C (2010) Age-related regional variations of the corpus callosum identified by diffusion tensor tractography. Neuroimage 52:20-31.

Lindenberg R, Zhu LL, Rüber T, Schlaug G (2012) Predicting functional motor potential in chronic stroke patients using diffusion tensor imaging. Hum Brain Mapp, in press.

Longa EZ, Weinstein PR, Carlson S, Cummins R (1989) Reversible middle cerebral artery occlusion without craniectomy in rats. Stroke 20:84-91.

Maslov S, Sneppen K (2002) Specificity and stability in topology of protein networks. Science 296:910-913.

Murphy TH, Corbett D (2009) Plasticity during stroke recovery: from synapse to behaviour. Nat Rev Neurosci 10:861-872.

Nichols T, Hayasaka S (2003) Controlling the familywise error rate in functional neuroimaging: a comparative review. Stat Methods Med Res 12:419-446.

Nudo RJ (2007) Postinfarct cortical plasticity and behavioral recovery. Stroke 38:840-845.

Pan W-J, Thompson G, Magnuson M, Majeed W, Jaeger D, Keilholz S (2011) Broadband local field potentials correlate with spontaneous fluctuations in functional magnetic resonance imaging signals in the rat somatosensory cortex under isoflurane anesthesia. Brain Connectivity 1:119-131.

Paxinos G, Watson C (2005) The rat brain in stereotaxic coordinates, Ed 5. Burlington, MA: Elsevier Academic.

Puig J, Pedraza S, Blasco G, Daunis-I-Estadella J, Prats A, Prados F, Boada I, Castellanos M, Sánchez-González J, Remollo S, Laguillo G, Quiles AM, Gómez E, Serena J (2010) Wallerian degeneration in the corticospinal tract evaluated by diffusion tensor imaging correlates with motor deficit 30 days after middle cerebral artery ischemic stroke. AJNR Am J Neuroradiol 31:1324-1330.

Radlinska B, Ghinani S, Leppert IR, Minuk J, Pike GB, Thiel A (2010) Diffusion tensor imaging, permanent pyramidal tract damage, and outcome in subcortical stroke. Neurology 75:1048-1054.

Schaechter JD, Fricker ZP, Perdue KL, Helmer KG, Vangel MG, Greve DN, Makris N (2009) Microstructural status of ipsilesional and contralesional corticospinal tract correlates with motor skill in chronic stroke patients. Hum Brain Mapp 30:3461-3474.

Smith SM, Johansen-Berg H, Jenkinson M, Rueckert D, Nichols TE, Miller KL, Robson MD, Jones DK, Klein JC, Bartsch AJ, Behrens TE (2007) Acquisition and voxelwise analysis of multi-subject diffusion data with tract-based spatial statistics. Nat Protoc 2:499-503.

Stam CJ, de Haan W, Daffertshofer A, Jones BF, Manshanden I, van Cappellen van Walsum AM, Montez T, Verbunt JP, de Munck JC, van Dijk BW, Berendse HW, Scheltens P (2009) Graph theoretical analysis of magnetoencephalographic functional connectivity in Alzheimer's disease. Brain 132:213-224.

Stroemer RP, Kent TA, Hulsebosch CE (1995) Neocortical neural sprouting, synaptogenesis, and behavioral recovery after neocortical infarction in rats. Stroke 26:2135-2144.

Thomalla G, Glauche V, Koch MA, Beaulieu C, Weiller C, Röther J (2004) 
Diffusion tensor imaging detects early Wallerian degeneration of the pyramidal tract after ischemic stroke. Neuroimage 22:1767-1774.

van der Zijden JP, Bouts MJ, Wu O, Roeling TA, Bleys RL, van der Toorn A, Dijkhuizen RM (2008a) Manganese-enhanced MRI of brain plasticity in relation to functional recovery after experimental stroke. J Cereb Blood Flow Metab 28:832-840.

van der Zijden JP, van der Toorn A, van der Marel K, Dijkhuizen RM (2008b) Longitudinal in vivo MRI of alterations in perilesional tissue after transient ischemic stroke in rats. Exp Neurol 212:207-212.

van Meer MP, Dijkhuizen RM (2010) Functional and structural MR imaging of brain reorganization after stroke. In: Brain repair after stroke (Cramer SC, Nudo RJ, eds), pp 57-66. Cambridge: Cambridge UP.

van Meer MP, van der Marel K, Otte WM, Berkelbach van der Sprenkel JW, Dijkhuizen RM (2010a) Correspondence between altered functional and structural connectivity in the contralesional sensorimotor cortex after unilateral stroke in rats: a combined resting-state functional MRI and manganeseenhanced MRI study. J Cereb Blood Flow Metab 30:1707-1711.

van Meer MP, van der Marel K, Wang K, Otte WM, El Bouazati S, Roeling TA, Viergever MA, Berkelbach van der Sprenkel JW, Dijkhuizen RM (2010b) Recovery of sensorimotor function after experimental stroke correlates with restoration of resting-state interhemispheric functional connectivity. J Neurosci 30:3964-3972.

Wang C, Stebbins GT, Nyenhuis DL, deToledo-Morrell L, Freels S, Gencheva E, Pedelty L, Sripathirathan K, Moseley ME, Turner DA, Gabrieli JD, Gorelick
PB (2006) Longitudinal changes in white matter following ischemic stroke: a three-year follow-up study. Neurobiol Aging 27:1827-1833.

Wang K, van Meer MP, van der Marel K, van der Toorn A, Xu L, Liu Y, Viergever MA, Jiang T, Dijkhuizen RM (2011) Temporal scaling properties and spatial synchronization of spontaneous blood oxygenation level-dependent (BOLD) signal fluctuations in rat sensorimotor network at different levels of isoflurane anesthesia. NMR Biomed 24:61-67.

Wang L, Yu C, Chen H, Qin W, He Y, Fan F, Zhang Y, Wang M, Li K, Zang Y, Woodward TS, Zhu C (2010) Dynamic functional reorganization of the motor execution network after stroke. Brain 133:1224-1238.

Ward BD (2000) Simultaneous inference for fMRI data. Retrieved February 29, 2012, from http://afni.nimh.nih.gov/afni/doc/manual/AlphaSim.

Weissenbacher A, Kasess C, Gerstl F, Lanzenberger R, Moser E, Windischberger C (2009) Correlations and anticorrelations in resting-state functional connectivity MRI: A quantitative comparison of preprocessing strategies. Neuroimage 47:1408-1416.

Westlye LT, Walhovd KB, Dale AM, Bjørnerud A, Due-Tønnessen P, Engvig A, Grydeland H, Tamnes CK, Ostby Y, Fjell AM (2010) Life-span changes of the human brain white matter: diffusion tensor imaging (DTI) and volumetry. Cereb Cortex 20:2055-2068.

Zuo XN, Kelly C, Adelstein JS, Klein DF, Castellanos FX, Milham MP (2010) Reliable intrinsic connectivity networks: test-retest evaluation using ICA and dual regression approach. Neuroimage 49:2163-2177. 